\title{
STRONG CONVERGENCE TO COMMON FIXED \\ POINTS OF NONEXPANSIVE MAPPINGS WITHOUT COMMUTATIVITY ASSUMPTION
}

\author{
YONGHONG YAO, RUDONG CHEN, AND HAIYUN ZHOU
}

Received 11 June 2006; Revised 27 July 2006; Accepted 2 August 2006

We introduce an iteration scheme for nonexpansive mappings in a Hilbert space and prove that the iteration converges strongly to common fixed points of the mappings without commutativity assumption.

Copyright (c) 2006 Yonghong Yao et al. This is an open access article distributed under the Creative Commons Attribution License, which permits unrestricted use, distribution, and reproduction in any medium, provided the original work is properly cited.

\section{Introduction}

Let $H$ be a real Hilbert space, and let $C$ be a nonempty closed convex subset of $H$. A mapping $T$ of $C$ into itself is said to be nonexpansive if

$$
\|T x-T y\| \leq\|x-y\|
$$

for each $x, y \in C$. For a mapping $T$ of $C$ into itself, we denote by $F(T)$ the set of fixed points of $T$. We also denote by $\mathbb{N}$ and $\mathbb{R}^{+}$the set of positive integers and nonnegative real numbers, respectively.

Baillon [1] proved the first nonlinear ergodic theorem. Let $C$ be a nonempty bounded convex closed subset of a Hilbert space $H$ and let $T$ be a nonexpansive mapping of $C$ into itself. Then, for an arbitrary $x \in C,\left\{(1 /(n+1)) \sum_{i=0}^{n} T^{i} x\right\}_{n=0}^{\infty}$ converges weakly to a fixed point of $T$. Wittmann [9] studied the following iteration scheme, which has first been considered by Halpern [3]:

$$
\begin{gathered}
x_{0}=x \in C, \\
x_{n+1}=\alpha_{n+1} x+\left(1-\alpha_{n+1}\right) T x_{n}, \quad n \geq 0,
\end{gathered}
$$

where a sequence $\left\{\alpha_{n}\right\}$ in $[0,1]$ is chosen so that $\lim _{n \rightarrow \infty} \alpha_{n}=0, \sum_{n=1}^{\infty} \alpha_{n}=\infty$, and $\sum_{n=1}^{\infty}$ $\left|\alpha_{n+1}-\alpha_{n}\right|<\infty$; see also Reich [7]. Wittmann proved that for any $x \in C$, the sequence 
2 Nonexpansive mappings without commutativity assumption

$\left\{x_{n}\right\}$ defined by (1.2) converges strongly to the unique element $P x \in F(T)$, where $P$ is the metric projection of $H$ onto $F(T)$.

Recall that two mappings $S$ and $T$ of $H$ into itself are called commutative if

$$
S T=T S,
$$

for all $x, y \in H$.

Recently, Shimizu and Takahashi [8] have first considered an iteration scheme for two commutative nonexpansive mappings $S$ and $T$ and proved that the iterations converge strongly to a common fixed point of $S$ and $T$. They obtained the following result.

Theorem 1.1 (see [8]). Let $H$ be a Hilbert space, and let $C$ be a nonempty closed convex subset of $H$. Let $S$ and $T$ be nonexpansive mappings of $C$ into itself such that $S T=T S$ and $F(S) \cap F(T)$ is nonempty. Suppose that $\left\{\alpha_{n}\right\}_{n=0}^{\infty} \subseteq[0,1]$ satisfies

(i) $\lim _{n \rightarrow \infty} \alpha_{n}=0$, and

(ii) $\sum_{n=0}^{\infty} \alpha_{n}=\infty$.

Then, for an arbitrary $x \in C$, the sequence $\left\{x_{n}\right\}_{n=0}^{\infty}$ generated by $x_{0}=x$ and

$$
x_{n+1}=\alpha_{n} x+\left(1-\alpha_{n}\right) \frac{2}{(n+1)(n+2)} \sum_{k=0}^{n} \sum_{i+j=k} S^{i} T^{j} x_{n}, \quad n \geq 0,
$$

converges strongly to a common fixed point $P x$ of $S$ and $T$, where $P$ is the metric projection of $H$ onto $F(S) \cap F(T)$.

Remark 1.2. At this point, we note that the authors have imposed the commutativity on the mappings $S$ and $T$. But there are many mappings, that do not satisfy $S T=T S$. For example, if $X=[-1 / 2,1 / 2]$, and $S$ and $T$ of $X$ into itself are defined by

$$
S=x^{2}, \quad T=\sin x,
$$

then $S T=\sin ^{2} x$, whereas $T S=\sin x^{2}$.

In this paper, we deal with the strong convergence to common fixed points of two nonexpansive mappings in a Hilbert space. We consider an iteration scheme for nonexpansive mappings without commutativity assumption and prove that the iterations converge strongly to a common fixed point of the mappings $T_{i}, i=1,2$.

\section{Preliminaries}

Let $C$ be a closed convex subset of a Hilbert space $H$ and let $S$ and $T$ be nonexpansive mappings of $C$ into itself. Then we consider the iteration scheme

$$
\begin{gathered}
x_{0}=x \in C, \\
x_{n+1}=\alpha_{n} x+\left(1-\alpha_{n}\right) \frac{2}{(n+1)(n+2)} \sum_{k=0}^{n} \sum_{i+j=k} S^{i} T^{j} y_{n}, \\
y_{n}=\beta_{n} x_{n}+\left(1-\beta_{n}\right) \frac{2}{(n+1)(n+2)} \sum_{k=0}^{n} \sum_{i+j=k} T^{i} S^{j} x_{n}, \quad n \geq 0,
\end{gathered}
$$


where $\left\{\alpha_{n}\right\}$ and $\left\{\beta_{n}\right\}$ are two sequences in $[0,1]$. We know that a Hilbert space $H$ satisfies Opial's condition [6], that is, if a sequence $\left\{x_{n}\right\}$ in $H$ converges weakly to an element $y$ of $H$ and $y \neq z$, then

$$
\liminf _{n \rightarrow \infty}\left\|x_{n}-y\right\|<\liminf _{n \rightarrow \infty}\left\|x_{n}-z\right\|
$$

In what follows, we will use $P_{C}$ to denote the metric projection from $H$ onto $C$; that is, for each $x \in H, P_{C}$ is the only point in $C$ with the property

$$
\left\|x-P_{C} x\right\|=\min _{u \in C}\|u-x\| .
$$

It is known that $P_{C}$ is nonexpansive and characterized by the following inequality: given $x \in H$ and $v \in H$, then $v=P_{C} x$ if and only if

$$
\langle x-v, v-y\rangle \geq 0, \quad y \in C .
$$

Now, we introduce several lemmas for our main result in this paper. The first lemma can be found in $[4,5,10]$.

Lemma 2.1. Assume $\left\{a_{n}\right\}$ is a sequence of nonnegative real numbers such that

$$
a_{n+1} \leq\left(1-\gamma_{n}\right) a_{n}+\delta_{n}
$$

where $\left\{\gamma_{n}\right\}$ is a sequence in $(0,1)$ and $\left\{\delta_{n}\right\}$ is a sequence such that

(1) $\sum_{n=1}^{\infty} \gamma_{n}=\infty$;

(2) $\lim \sup _{n \rightarrow \infty} \delta_{n} / \gamma_{n} \leq 0$ or $\sum_{n=1}^{\infty}\left|\delta_{n}\right|<\infty$.

Then $\lim _{n \rightarrow \infty} a_{n}=0$.

Lemma 2.2. Let $C$ be a nonempty bounded closed convex subset of a Hilbert $H$, and let $S, T$ be nonexpansive mappings of $C$ into itself. For $x \in C$ and $n \in \mathbb{N} \cup\{0\}$, put

$$
\begin{aligned}
G_{n}(x) & =\frac{2}{(n+1)(n+2)} \sum_{k=0}^{n} \sum_{i+j=k} S^{i} T^{j} x, \\
\bar{G}_{n}(x) & =\frac{2}{(n+1)(n+2)} \sum_{k=0}^{n} \sum_{i+j=k} T^{i} S^{j} x .
\end{aligned}
$$

Then

$$
\begin{aligned}
& \limsup _{n \rightarrow \infty}\left\|G_{x \in C}(x)-S G_{n}(x)\right\|=0, \\
& \lim _{n \rightarrow \infty} \sup _{x \in C}\left\|\bar{G}_{n}(x)-T \bar{G}_{n}(x)\right\|=0 .
\end{aligned}
$$




\section{Nonexpansive mappings without commutativity assumption}

Proof. We first prove $\lim _{n \rightarrow \infty} \sup _{x \in C}\left\|G_{n}(x)-S G_{n}(x)\right\|=0$.

By an idea in [2], for $\left\{x_{i, j}\right\}_{i, j=0}^{\infty},\left\{\bar{x}_{i, j}\right\}_{i, j=0}^{\infty} \subseteq C$ and $z_{n}=\left(1 / l_{n}\right) \sum_{k=0}^{n} \sum_{i+j=k} x_{i, j}, \bar{z}_{n}=$ $\left(1 / l_{n}\right) \sum_{k=0}^{n} \sum_{i+j=k} \bar{x}_{i, j} \in C$, with $l_{n}=(n+1)(n+2) / 2$, we have

$$
\left\|z_{n}-v\right\|^{2}=\frac{1}{l_{n}} \sum_{k=0}^{n} \sum_{i+j=k}\left\|x_{i, j}-v\right\|^{2}-\frac{1}{l_{n}} \sum_{k=0}^{n} \sum_{i+j=k}\left\|x_{i, j}-z_{n}\right\|^{2}
$$

for each $v \in H$. For $x \in C$, put $x_{i, j}=S^{i} T^{j} x, \bar{x}_{i, j}=T^{i} S^{j} x$ and $v=S z_{n}, \bar{v}=T \bar{z}_{n}$. Then, we have

$$
\begin{aligned}
& \left\|G_{n}(x)-S G_{n}(x)\right\|^{2}=\frac{1}{l_{n}} \sum_{k=0}^{n} \sum_{i+j=k}\left\|S^{i} T^{j} x-S z_{n}\right\|^{2}-\frac{1}{l_{n}} \sum_{k=0}^{n} \sum_{i+j=k}\left\|S^{i} T^{j} x-z_{n}\right\|^{2} \\
& =\frac{1}{l_{n}} \sum_{k=0}^{n}\left\|T^{k} x-S z_{n}\right\|^{2}+\frac{1}{l_{n}} \sum_{k=1}^{n} \sum_{i+j=k, i \geq 1}\left\|S^{i} T^{j} x-S z_{n}\right\|^{2} \\
& -\frac{1}{l_{n}} \sum_{k=0}^{n} \sum_{i+j=k}\left\|S^{i} T^{j} x-z_{n}\right\|^{2} \\
& \leq \frac{1}{l_{n}} \sum_{k=0}^{n}\left\|T^{k} x-S z_{n}\right\|^{2}+\frac{1}{l_{n}} \sum_{k=1}^{n} \sum_{i+j=k, i \geq 1}\left\|S^{i-1} T^{j} x-z_{n}\right\|^{2} \\
& -\frac{1}{l_{n}} \sum_{k=0}^{n} \sum_{i+j=k}\left\|S^{i} T^{j} x-z_{n}\right\|^{2} \\
& =\frac{1}{l_{n}} \sum_{k=0}^{n}\left\|T^{k} x-S z_{n}\right\|^{2}+\frac{1}{l_{n}} \sum_{k=0}^{n-1} \sum_{i+j=k}\left\|S^{i} T^{j} x-z_{n}\right\|^{2} \\
& -\frac{1}{l_{n}} \sum_{k=0}^{n} \sum_{i+j=k}\left\|S^{i} T^{j} x-z_{n}\right\|^{2} \\
& =\frac{1}{l_{n}} \sum_{k=0}^{n}\left\|T^{k} x-S z_{n}\right\|^{2}-\frac{1}{l_{n}} \sum_{i+j=n}\left\|S^{i} T^{j} x-z_{n}\right\|^{2} \\
& \leq \frac{1}{l_{n}} \sum_{k=0}^{n}\left\|T^{k} x-S z_{n}\right\|^{2} \leq \frac{2}{n+2}\{\operatorname{diam}(C)\}^{2},
\end{aligned}
$$

where $\operatorname{diam}(C)$ is the diameter of $C$. So, we have, for each $n \in \mathbb{N} \cup\{0\}$,

$$
\sup _{x \in C}\left\|G_{n}(x)-S G_{n}(x)\right\|^{2} \leq \frac{2}{n+2}\{\operatorname{diam}(C)\}^{2},
$$

and hence

$$
\lim _{n \rightarrow \infty} \sup _{x \in C}\left\|G_{n}(x)-S G_{n}(x)\right\|=0 .
$$


Similarly, we have

$$
\lim _{n \rightarrow \infty} \sup _{x \in C}\left\|\bar{G}_{n}(x)-T \bar{G}_{n}(x)\right\|=0 .
$$

\section{Convergence theorem}

Now we can prove a strong convergence theorem in a Hilbert space.

Theorem 3.1. Let $H$ be a Hilbert space, and let $C$ be a nonempty closed convex subset of $H$. Let $S$ and $T$ be nonexpansive mappings of $C$ into itself such that $F(S) \cap F(T)$ is nonempty. Suppose that $\left\{\alpha_{n}\right\}_{n=0}^{\infty}$ and $\left\{\beta_{n}\right\}_{n=1}^{\infty}$ are two sequences in $[0,1]$ satisfying the following conditions:

(i) $\lim _{n \rightarrow \infty} \alpha_{n}=0$, and

(ii) $\sum_{n=0}^{\infty} \alpha_{n}=\infty$.

For an arbitrary $x \in C$, the sequence $\left\{x_{n}\right\}_{n=0}^{\infty}$ is generated by $x_{0}=x$ and

$$
\begin{gathered}
x_{n+1}=\alpha_{n} x+\left(1-\alpha_{n}\right) \frac{2}{(n+1)(n+2)} \sum_{k=0}^{n} \sum_{i+j=k} S^{i} T^{j} y_{n}, \\
y_{n}=\beta_{n} x_{n}+\left(1-\beta_{n}\right) \frac{2}{(n+1)(n+2)} \sum_{k=0}^{n} \sum_{i+j=k} T^{i} S^{j} x_{n}, \quad n \geq 0 .
\end{gathered}
$$

Let

$$
z_{n}=\frac{2}{(n+1)(n+2)} \sum_{k=0}^{n} \sum_{i+j=k} S^{i} T^{j} y_{n}, \quad \bar{z}_{n}=\frac{2}{(n+1)(n+2)} \sum_{k=0}^{n} \sum_{i+j=k} T^{i} S^{j} x_{n}
$$

for each $n \in \mathbb{N} \cup\{0\}$. If there exist subsequences $\left\{z_{n_{i}}\right\}_{i=0}^{\infty}$ of $\left\{z_{n}\right\}_{n=0}^{\infty}$ and $\left\{\bar{z}_{n_{j}}\right\}_{j=0}^{\infty}$ of $\left\{\bar{z}_{n}\right\}_{n=0}^{\infty}$, respectively, which converge weakly to some common point $z$ in some bounded subset $D$ of $C$, then the sequence $\left\{x_{n}\right\}_{n=0}^{\infty}$ defined by (3.1) converges strongly to $P_{F(S) \cap F(T)} x$.

Proof. Let $x \in C$ and $w \in F(S) \bigcap F(T)$. Putting $r=\|x-w\|$, then the set

$$
D=\{y \in H:\|y-w\| \leq r\} \cap C
$$

is a nonempty bounded closed convex subset of $C$ which is $S$ - and $T$-invariant and contains $x_{0}=x$. So we may assume, without loss of generality, that $S$ and $T$ are the mappings of $D$ into itself. Since $P$ is the metric projection of $H$ onto $F(S) \cap F(T)$, we have

$$
\langle y-P x, x-P x\rangle \leq 0
$$

for each $y \in F(S) \bigcap F(T)$. 
6 Nonexpansive mappings without commutativity assumption

From (3.4), we have

$$
\limsup _{n \rightarrow \infty}\left\langle z_{n}-P x, x-P x\right\rangle \leq 0, \quad \quad \limsup _{n \rightarrow \infty}\left\langle\bar{z}_{n}-P x, x-P x\right\rangle \leq 0 .
$$

In fact, assume that, there exist two positive real numbers $r_{0}$ and $r_{1}$ such that

$$
\limsup _{n \rightarrow \infty}\left\langle z_{n}-P x, x-P x\right\rangle>r_{0}, \quad \limsup _{n \rightarrow \infty}\left\langle\bar{z}_{n}-P x, x-P x\right\rangle>r_{1} .
$$

Since $\left\{z_{n}\right\}_{n=0}^{\infty}$ and $\left\{\bar{z}_{n}\right\}_{n=0}^{\infty} \subseteq D$ are bounded, from (3.6), there exist subsequences $\left\{z_{n_{i}}\right\}_{i=0}^{\infty}$ of $\left\{z_{n}\right\}_{n=0}^{\infty}$ and $\left\{\bar{z}_{n_{j}}\right\}_{j=0}^{\infty}$ of $\left\{\bar{z}_{n}\right\}_{n=0}^{\infty}$, respectively, such that

$$
\begin{gathered}
\limsup _{n \rightarrow \infty}\left\langle z_{n}-P x, x-P x\right\rangle=\lim _{i \rightarrow \infty}\left\langle z_{n_{i}}-P x, x-P x\right\rangle>r_{0}, \\
\limsup _{n \rightarrow \infty}\left\langle\bar{z}_{n}-P x, x-P x\right\rangle=\lim _{j \rightarrow \infty}\left\langle\bar{z}_{n_{j}}-P x, x-P x\right\rangle>r_{1} .
\end{gathered}
$$

By the assumption, we know that $\left\{z_{n_{i}}\right\}_{i=0}^{\infty}$ and $\left\{\bar{z}_{n_{j}}\right\}_{j=0}^{\infty}$ converge weakly to some common point $z \in D$. Thus from Lemma 2.2 and Opial's condition, we have $z \in F(S) \cap F(T)$. In fact, if $z \neq S z$, we have

$$
\begin{aligned}
\liminf _{i \rightarrow \infty}\left\|z_{n_{i}}-z\right\| & <\liminf _{i \rightarrow \infty}\left\|z_{n_{i}}-S z\right\| \\
& \leq \liminf _{i \rightarrow \infty}\left(\left\|z_{n_{i}}-S z_{n_{i}}\right\|+\left\|S z_{n_{i}}-S z\right\|\right) \\
& \leq \liminf _{i \rightarrow \infty}\left\|z_{n_{i}}-z\right\| .
\end{aligned}
$$

This is a contradiction. Therefore, we have $z=S z$.

Similarly, we have $z=T z$. So, we have

$$
\langle z-P x, x-P x\rangle \leq 0
$$

On the other hand, since $\left\{z_{n_{i}}\right\}$ converges weakly to $z$, we obtain

$$
\langle z-P x, x-P x\rangle \geq r_{0} .
$$

This is a contradiction. Hence, we have

$$
\limsup _{n \rightarrow \infty}\left\langle z_{n}-P x, x-P x\right\rangle \leq 0, \quad \quad \limsup _{n \rightarrow \infty}\left\langle\bar{z}_{n}-P x, x-P x\right\rangle \leq 0 .
$$


Since

$$
\begin{aligned}
\left\|\bar{z}_{n}-P x\right\| \leq & \left.\frac{2}{(n+1)(n+2)} \sum_{k=0}^{n} \sum_{i+j=k}\left\|T^{i} S^{j} x_{n}-P x\right\|\right\}^{2} \\
\leq & \left\{\frac{2}{(n+1)(n+2)} \sum_{k=0}^{n} \sum_{i+j=k}\left\|x_{n}-P x\right\|\right\}^{2}=\left\|x_{n}-P x\right\|^{2}, \\
\left\|y_{n}-P x\right\|^{2}= & \left\|\beta_{n} x_{n}+\left(1-\beta_{n}\right) \bar{z}_{n}-P x\right\|^{2} \\
= & \left\|\beta_{n}\left(x_{n}-P x\right)+\left(1-\beta_{n}\right)\left(\bar{z}_{n}-P x\right)\right\|^{2} \\
= & \beta_{n}^{2}\left\|x_{n}-P x\right\|^{2}+2 \beta_{n}\left(1-\beta_{n}\right)\left(x_{n}-P x, \bar{z}_{n}-P x\right)+\left(1-\beta_{n}\right)^{2}\left\|\bar{z}_{n}-P x\right\|^{2} \\
\leq & \beta_{n}^{2}\left\|x_{n}-P x\right\|^{2}+2 \beta_{n}\left(1-\beta_{n}\right) \frac{\left\|x_{n}-P x\right\|^{2}+\left\|\bar{z}_{n}-P x\right\|^{2}}{2} \\
& +\left(1-\beta_{n}\right)^{2}\left\|\bar{z}_{n}-P x\right\|^{2} \leq\left\|x_{n}-P x\right\|^{2} .
\end{aligned}
$$

Then, we have

$$
\begin{aligned}
\left\|x_{n+1}-P x\right\|^{2}= & \left\|\alpha_{n} x+\left(1-\alpha_{n}\right) z_{n}-P x\right\|^{2} \\
= & \alpha_{n}^{2}\|x-P x\|^{2}+\left(1-\alpha_{n}\right)^{2}\left\|z_{n}-P x\right\|^{2}+2 \alpha_{n}\left(1-\alpha_{n}\right)\left\langle z_{n}-P x, x-P x\right\rangle \\
\leq & \left(1-\alpha_{n}\right)^{2}\left\{\frac{2}{(n+1)(n+2)} \sum_{k=0}^{n} \sum_{i+j=k}\left\|S^{i} T^{j} y_{n}-P x\right\|\right\}^{2} \\
& +\alpha_{n}^{2}\|x-P x\|^{2}+2 \alpha_{n}\left(1-\alpha_{n}\right)\left\langle z_{n}-P x, x-P x\right\rangle \\
\leq & \left(1-\alpha_{n}\right)^{2}\left\{\frac{2}{(n+1)(n+2)} \sum_{k=0}^{n} \sum_{i+j=k}\left\|y_{n}-P x\right\|\right\}^{2} \\
& +\alpha_{n}^{2}\|x-P x\|^{2}+2 \alpha_{n}\left(1-\alpha_{n}\right)\left\langle z_{n}-P x, x-P x\right\rangle \\
= & \left(1-\alpha_{n}\right)^{2}\left\|y_{n}-P x\right\|^{2}+\alpha_{n}^{2}\|x-P x\|^{2}+2 \alpha_{n}\left(1-\alpha_{n}\right)\left\langle z_{n}-P x, x-P x\right\rangle \\
\leq & \left(1-\alpha_{n}\right)\left\|x_{n}-P x\right\|^{2}+\alpha_{n}\left\{\alpha_{n}\|x-P x\|^{2}+2\left(1-\alpha_{n}\right)\left\langle z_{n}-P x, x-P x\right\rangle\right\} .
\end{aligned}
$$

Putting $a_{n}=\left\|x_{n}-P x\right\|^{2}$, from (3.13), we have

$$
a_{n+1} \leq\left(1-\alpha_{n}\right) a_{n}+\delta_{n}
$$

where $\delta_{n}=\alpha_{n}\left\{\alpha_{n}\|x-P x\|^{2}+2\left(1-\alpha_{n}\right)\left\langle z_{n}-P x, x-P x\right\rangle\right\}$. 
It is easily seen that

$$
\limsup _{n \rightarrow \infty} \delta_{n} / \alpha_{n}=\limsup _{n \rightarrow \infty}\left\{\alpha_{n}\|x-P x\|^{2}+2\left(1-\alpha_{n}\right)\left\langle z_{n}-P x, x-P x\right\rangle\right\} \leq 0 .
$$

Now applying Lemma 2.1 with (3.15) to (3.14) concludes that $\left\|x_{n}-P x\right\| \rightarrow 0$ as $n \rightarrow \infty$. This completes the proof.

\section{References}

[1] J.-B. Baillon, Un théorème de type ergodique pour les contractions non linéaires dans un espace de Hilbert, Comptes Rendus de l'Académie des Sciences de Paris, Série. A-B 280 (1975), no. 22, A1511-A1514.

[2] H. Brézis and F. E. Browder, Nonlinear ergodic theorems, Bulletin of the American Mathematical Society 82 (1976), no. 6, 959-961.

[3] B. Halpern, Fixed points of nonexpanding maps, Bulletin of the American Mathematical Society 73 (1967), 957-961.

[4] J. S. Jung, Viscosity approximation methods for a family of finite nonexpansive mappings in Banach spaces, Nonlinear Analysis 64 (2006), no. 11, 2536-2552.

[5] P.-E. Maingé, Viscosity methods for zeroes of accretive operators, Journal of Approximation Theory 140 (2006), no. 2, 127-140.

[6] Z. Opial, Weak convergence of the sequence of successive approximations for nonexpansive mappings, Bulletin of the American Mathematical Society 73 (1967), 591-597.

[7] S. Reich, Some problems and results in fixed point theory, Topological Methods in Nonlinear Functional Analysis (Toronto, Ont., 1982), Contemp. Math., vol. 21, American Mathematical Society, Rhode Island, 1983, pp. 179-187.

[8] T. Shimizu and W. Takahashi, Strong convergence to common fixed points of families of nonexpansive mappings, Journal of Mathematical Analysis and Applications 211 (1997), no. 1, 71-83.

[9] R. Wittmann, Approximation of fixed points of nonexpansive mappings, Archiv der Mathematik 58 (1992), no. 5, 486-491.

[10] H.-K. Xu, Viscosity approximation methods for nonexpansive mappings, Journal of Mathematical Analysis and Applications 298 (2004), no. 1, 279-291.

Yonghong Yao: Department of Mathematics, Tianjin Polytechnic University, Tianjin 300160, China E-mail address: yuyanrong@tjpu.edu.cn

Rudong Chen: Department of Mathematics, Tianjin Polytechnic University, Tianjin 300160, China E-mail address: chenrd@tjpu.edu.cn

Haiyun Zhou: Department of Mathematics, Shijiazhuang Mechanical Engineering College, Shijiazhuang 050003, China

E-mail address: witman66@yahoo.com.cn 